$\begin{array}{lcl}\text { Bentham OPEN } \\ \text { CrossMark } & \text { Content list available at: www.benthamopen.com/MEDJ/ } \\ \text { DOI: } 10.2174 / 1874220301603010230 & \text { Open } \\ \text { Medicine } \\ \text { lournal }\end{array}$

RESEARCH ARTICLE

\title{
Implementing Case Management within a Large Medicolegal Death Investigation Agency
}

\author{
Stacy A. Drake ${ }^{1, *}$, Sherhonda Harper ${ }^{2}$ and Antoinette Hudson ${ }^{2}$ \\ ${ }^{I}$ The University of Texas Health Science Center at Houston, School of Nursing Department of Systems, 6901 Bertner \\ Ave., \#748, Houston, Texas 77030, United States \\ ${ }^{2}$ Harris County Institute of Forensic Sciences, 1885 Old Spanish Trail, Houston, Texas 77054, United States
}

Received: September 16, 2015

Revised: October 07, 2015

Accepted: October 27, 2015

\begin{abstract}
Medicolegal death investigation agencies must provide timely final autopsy reports in order to meet minimum accreditation standards. To ensure a timely turn around, the principles of case management were introduced into an agency with a large metropolitan jurisdiction. Forensic autopsies are typically complex and the associated ancillary studies often include forensic toxicology along with various specialty consults. Beginning in 2013, a forensic case management service was initiated to aid forensic pathologists in reducing report turnaround time. Despite increasing number of cases in 2014, the agency was able to maintain the accreditation standard of $90 \%$ turn around within 90 days. The case management service required process improvement, technology to track and trend, and increased interdisciplinary collaboration. The implementation of a case management system within the forensic autopsy service can improve processes to reduce report turnaround times.
\end{abstract}

Keywords: Implementing case management, Interdisciplinary collaboration, Medicolegal death, Turnaround time.

\section{INTRODUCTION}

A responsibility of medicolegal death investigations (MLDI) agencies is the investigation of sudden and unexpected deaths which often include the performance of an autopsy. The autopsy report is important as it provides information presented to families, justice systems, and vital statistics agencies. Both the College of American Pathologists and the National Association of Medical Examiners (NAME) provide accreditation guidelines. One performance measure in both sets of accreditation guidelines is turnaround time (TAT) of final autopsy reports within a reasonable time frame. The essential (phase 2) requirement for NAME TAT is completion of at least $90 \%$ of autopsy reports within 90 calendar days from the date of autopsy. NAME also provides a phase 1 deficiency for agencies where $90 \%$ of autopsy reports are not completed within 60 days. Many large offices (Harris County personal communication) consider the 60 day turnaround time to be a "best practice" standard, but typically not feasible in a sustained way. This is particularly true in offices where workload approaches maximum allowed NAME standards, and in which violent and other non-natural deaths comprise a disproportionately large percentage of the overall caseload. Workload standards under NAME guidelines are that pathologists should perform no more than 250 autopsies per year and are not to exceed 325 autopsies per year [1]. College of American Pathologist TAT guidelines is 30 days for routine cases and 3 months for complicated $[2]$.

Turnaround time is a significant performance measure in assessing efficiency and timeliness of autopsy services provided. Case volume, autopsy complexity, staffing, and workflow processes influence turnaround time [2 - 4]. In addition, forensic autopsies often have various layers of complexity due to required subspecialty consults (e.g. neuropathology, pediatric pathology, cardiac pathology, or anthropology; detailed toxicology; and investigation from

\footnotetext{
* Address correspondence to this author at The University of Texas Health Science Center at Houston, School of Nursing Department of Systems, 6901 Bertner Ave., \#748, Houston, Texas 77030; United States; Tel: 713-500-2113; Fax: 713-500-2142; E-mail: Stacy.A.Drake@uth.tmc.edu
} 
outside agencies). Pressure to maintain acceptable TAT rates can result in negative consequences, such as low staff morale, staff burnout, and decreased retention [5]. In healthcare settings, nurses have utilized processes of case management to coordinate health and human services for over a century. Regardless of the setting, principles of case management remain the same. That is, case management models serve to coordinate complex, fragmented services, while controlling costs, to meet the needs of clients [6].

According to the Case Management Society, a premise of case management is that all constituents benefit when desired outcomes are achieved [7]. A case manager within a clinical setting identifies appropriate providers and facilitates a continuum of services in as cost effective and timely manner as possible. Case management has the following five fundamental sequential activities: 1) assess the need, 2) develop a plan, 3) link services, 4) monitor progress, and 5) communicate as needed [7]. Case management services work optimally when the environment permits direct communication among the case manager, customers, and service personnel.

\section{MATERIALS AND METHODOLOGY}

The purpose of the project was to assess how implementation of a forensic nurse case management program would impact TAT within a large MLDI agency located in the third largest county in the United States. The MLDI agency investigates approximately 10,000 of over 31,000 deaths annually in that county where over 4,000 require a forensic pathologist to determine the cause and manner of death [7]. The MLDI agency is NAME accredited and has 15 forensic pathologists, a full-time forensic neuropathologist, three forensic anthropologists, and full crime laboratory service. The agency has the capacity to train two forensic pathology fellows each year. Additionally, the agency serves as a resource to medical schools and hosts approximately 30 medical students per year, up to three residents per month, and interns, including international trainees.

The forensic nurse specialist program includes two forensic nurse specialists (FNS) serving as forensic case managers. The FNSs were administratively members of the pathology division, were physically located in the pathology department (in the vicinity of pathologists' offices and file records storage) and worked collaboratively with senior administration. Based on assessment of needs, the FNS role focused on processes to improve TAT and included assessing existing policies, procedures, and guidelines impacting TAT; establishing inter-professional collaborations among information technology, pathology, histology, and investigations divisions; and fostering improved communication among students, residents, and fellows. The case management team effort was initiated in January 2013 with two FNSs as case managers. By January 2014, process changes were implemented.

\section{Pre-case Management}

Pre-case management was the assessment, collaboration, and plan design phase. Protocols were examined, redundancy reduced, and format as well as explicit content simplified. A plan for implementing initiatives was designed based on input from collaborative efforts and developed to include electronic linkage of services and permit case monitoring for eliciting timely feedback. A typical weekly routine for FNS case managers included the following:

1. Attendance at daily meetings with pathologists for case review and assignment.

2. Attendance at weekly peer review of cases outstanding over 80 days, where pathologists were notified of the cases prior to the meeting.

3. Work with pathologists to notify forensic investigators via face to face communication or personal email to conduct any required follow-up.

4. Acting as liaison directly with laboratories (inside the agency and outside the agency), with police investigators, and with emergency medical agencies to obtain results and necessary investigative follow-up.

5. Acting as liaison directly with the public health department regarding reportable disease.

\section{Case Management Initiatives}

Process improvement strategies employed by FNS case managers included the following:

1. Reduced duplication of services within the pathology division, then monitoring and tracking cases to ensure timely follow-up.

2. Initiation of a case management spreadsheet and daily case status updates within a case management software. Updated information included information on pending toxicology reports. 
3. At the daily case review meeting, identification of immediate case management needs, including receipt of radiology reports to determine whether an autopsy or an external examination is required; documentation of any additional necessary follow up such as obtaining specific medical records, or contacting families for specific additional information.

4. Conducting follow-up with public health, medical, and other providers regarding reportable infectious disease deaths, suicide after discharge from healthcare settings, or other healthcare quality issues that impact community providers.

5. Collaboration with other laboratories or agencies within or outside the office (e.g. toxicology laboratory, investigations division, law enforcement agencies) or administrators within medical records to maximize efficiency in obtaining case outcomes.

6. Stratification of pending cases based upon stratified timeframes in order to disseminate weekly reports to pathologists to alert them of current pending and incomplete cases.

7. Maintaining and documentation of accreditation requirements within the pathology division to monitor TAT practices.

\section{Case Selection}

To determine if the criterion of $90 \%$ completion of autopsy reports occurred within 90 calendar days from the time of the autopsy, cases were retrospectively selected from medical records for the years 2011 and 2012 to determine the baseline time frame for completion of final autopsy reports. Forensic autopsies conducted in the years 2011 and 2012 were reviewed at days 60 and 90 to determine the yearly number of examinations, consults, and number of full-time forensic pathologists employed at the time. This process continued in the year 2013, when the case management team was in the pre-management phase. At this time, case management responsibilities focused on observing daily routines and attending collaborative meetings regarding proposed case management initiatives. The 60 and 90 day review and continued throughout the year 2014, when case management initiatives were implemented.

\section{Outcomes}

The total number of cases for each year is shown in Table 1. Although 2011 baseline shows $91 \%$ completion of final autopsy reports $90 \%$ of cases signed and completed in 2014 included an additional 386 cases. However, the agency had two more fulltime pathologists in 2013 and 2014. For the year 2014, TAT was achieved.

Table 1. Turn around times for years 2011-2014.

\begin{tabular}{|c|c|c|c|c|c|}
\hline Year & Total Cases & Cases signed within 60 days & Cases signed within 90 days & No. Fellows & $\begin{array}{l}\text { No. Full-time } \\
\text { Pathologists }\end{array}$ \\
\hline 2011 & 3143 & $79 \%$ & $91 \%$ & 2 & 13 \\
\hline 2012 & 3264 & $76 \%$ & $86 \%$ & 1 & 14 \\
\hline *2013 & 3428 & $75 \%$ & $85 \%$ & 1.5 & 15 \\
\hline$* * 2014$ & 3529 & $74 \%$ & $90 \%$ & 1 & 15 \\
\hline
\end{tabular}

*Case Management team initiated ** Implementation of process changes

\section{DISCUSSION}

The pathology service line in 2014 made an administrative decision to focus on the realistic goal of completing $90 \%$ of final autopsy reports within 90 days. This decision was based on availability of better technology to aid in determining cause of death (e.g. histology staining and increased toxicology and genetic testing). Also, NAME accreditation standards recognized the complexity of forensic cases and executed a less significant accreditation penalty for 60 days than that of 90 days [1]. The additional complexities for forensic cases included infant deaths, specialty consults, an increasing number of deaths requiring extensive forensic toxicology, and multiple gunshot wound or stabbing deaths [9]. The yearly average of infant deaths (0-365 days) is 115 in Harris County, and these deaths require extensive histology, investigation, a neuropathy and probable pediatric pathology consults [8]. The number of anthropology consults is 160 per year (with an additional anthropology autopsy consult of 50 at the autopsy table). In addition, the county averages 200 neuropathology and 100 cardiopathology consults [8]. Average number of homicides is 398 with an average of over 400 hours of courtroom time per year. Toxicology is performed on almost all deaths requiring an autopsy. Therefore, the number of details required in the autopsy report makes it challenging to complete each report in a timely manner. 
After the first year of case management implementation, the case managers achieved the overall outcome goal of improvement in TAT despite the increased number of cases. In 2014, case autopsies completed and signed within 90 days increased from $85 \%$ to $90 \%$. The case management program initiatives, through assessing, evaluating, and refining processes, changed the flow and operation of pathology services. The case managers transitioned as active collaborators within the pathology department to essential medicolegal death investigation team members. The pathologists have the ability to focus more on completing the autopsy report and less time spend on following up on cases since the implementation of case management. The pathologists have expressed the benefits while adjusting to the new relationship between case management and forensic pathologists.

\section{CONCLUSION}

Case management can provide significant benefits for medicolegal death investigations. The emerging role of case managers within medicolegal death investigation supports and promotes optimization of outcomes. The forensic investigators managed triaging incoming deaths, the pathologists focused on autopsies, and the case managers coordinated services, conducted follow-up with families, made calls to law enforcement and health care facilities for information, and managed records requests. The FNS functioned as major collaborators within the team in promoting process improvements and coordination with the investigation division. A delay in issuing timely autopsy reports could ultimately lead to frustration for families, delays in relation to the justice system, a decline in interest among the medical communities, increase in pending death certificates, negative reviews related to an agency's performance, and issues with accreditation $[3,5]$. The forensic case management model is easily adaptable within the medicolegal death investigative setting, and the use of a process improvement model provides agencies with a method of ensuring TAT standards and quality are sustained.

\section{CONFLICT OF INTEREST}

The authors confirm that this article content has no conflict of interest.

\section{ACKNOWLEDGEMENTS}

Declared none.

\section{REFERENCES}

[1] National Association of Medical Examiners. Inspection and Accreditation Standards. Available at: https://netforum.avectra.com/public/temp/ ClientImages/NAME/3c58b363-0fa6-42cc-9f71-d2c5c0790cb1.pdf [Accessed May 2015];

[2] Baker PB. Communication of Autopsy Reports. Available at: http://www.cap.org/apps/docs/pathology_reporting/Communicate AutopsyResults.pdf [Accessed August 2015];

[3] Baker PB, Zarbo RJ, Howanitz PJ. Quality assurance of autopsy face sheet reporting, final autopsy report turnaround time, and autopsy rates: a College of American Pathologists Q-Probes study of 10003 autopsies from 418 institutions. Arch Pathol Lab Med 1996; $120(11)$ : 1003-8. [PMID: 12049099]

[4] Smith MT, Garvin AJ. mhatomic pathology turnaround times. Use and abuse. Am J Clin Pathol 1996; 106(4)(Suppl. 1): S70-3. [PMID: 8853060]

[5] Siebert JR. Increasing the efficiency of autopsy reporting. Arch Pathol Lab Med 2009; 133(12): $1932-7$. [PMID: 19961247]

[6] Kersbergen AL. Case management: a rich history of coordinating care to control costs. Nurs Outlook 1996; 44(4): 169-72. [http://dx.doi.org/10.1016/S0029-6554(96)80037-6] [PMID: 8871998]

[7] Case Management Society. Available at: http://www.cmsa.org/CMSA/WhoWeAre/tabid/222/default.aspx [Accessed May 2015];

[8] Harris County Institute of Forensic Sciences. Available at: http://www.harriscountytx.gov/ifs/ [Accessed June 2015];

[9] Tormos LM, Schandl CA, Batalis NI, Presnell SE. Five-day turnaround time for hospital medical autopsies: A five-year experience. Acad Forensic Pathol 2015; 5(2): 233-9.

C) Drake et al.; Licensee Bentham Open

This is an open access article licensed under the terms of the Creative Commons Attribution-Non-Commercial 4.0 International Public License (CC BY-NC 4.0) (https://creativecommons.org/licenses/by-nc/4.0/legalcode), which permits unrestricted, non-commercial use, distribution and reproduction in any medium, provided the work is properly cited. 